\title{
NOTE ON SOME ELEMENTARY PROPERTIES OF POLYNOMIALS
}

\section{P. ERDÖS}

In a previous paper T. Grünwald ${ }^{1}$ and I proved that if $f(x)$ is a polynomial of degree $n \geqq 2$ and satisfies the following conditions:

$$
\begin{aligned}
& \text { all roots of } f(x) \text { are real, } f(-1)=f(+1)=0 \\
& f(x) \neq 0 \text { for }-1<x<1, \max _{-1<x<1} f(x)=1
\end{aligned}
$$

then

$$
\int_{-1}^{+1} f(x) \leqq \frac{4}{3}
$$

Equality occurs only for $f(x)=1-x^{2}$.

This result can be generalized as follows: Suppose $f(x)$ satisfies (1) and let $f(a)=f(b)=d \leqq 1,-1<a<b<1$; then

$$
b-a \leqq 2(1-d)^{1 / 2} \text {. }
$$

Again equality occurs only for $f(x)=1-x^{2}$. It is clear that (2) follows from (3) by integration with respect to $d$.

PROOF. Instead of (3) we prove the following slightly more general result: Let $f(x)$ satisfy (1), and determine the greatest positive constant $c_{f}$ such that

$$
f(a) f\left(a+c_{f}\right)=d^{2}, \quad-1<a<a+c_{f}<1
$$

then

$$
c_{f} \leqq 2(1-d)^{1 / 2}
$$

Equality holds only for $f(x)=1-x^{2}, a=-(1-d)^{1 / 2}$.

Suppose there exists a polynomial of degree $n>2$ satisfying (1) with $c_{f} \geqq 2(1-d)^{1 / 2}$; then we will prove that there exists a polynomial of degree $n-1$ with $c_{f}>2(1-d)^{1 / 2}$; and this proves (4) since it is easy to prove that (4) is satisfied for polynomials of second degree, that is, for $1-x^{2}$.

Denote the roots of $f(x)$ by $x_{1}=-1, x_{2}=1, x_{3}, \cdots, x_{n}$ and suppose first that for $i>2$ the $x_{i}$ are not all of the same sign. Let $x_{n}$ be the largest positive root and $x_{n-1}$ the smallest negative root, and denote by $y$ the root of $f^{\prime}(x)$ in $(-1,+1)$. Consider the polynomial of degree $n$

\footnotetext{
1 Annals of Mathematics, (2), vol. 40 (1939), pp. 537-548.
} 


$$
\phi(x)=c \frac{f(x)(x-y)^{2}}{\left(x-x_{n}\right)\left(x-x_{n-1}\right)},
$$

where we choose $c$ so that $\phi(x) \geqq 0$ for $-1 \leqq x \leqq 1$. Then it is easy to see that for large $x, \phi(x)$ and $f(x)$ have opposite signs. Thus their leading coefficients have opposite signs. Hence it is possible to choose $c$ such that the polynomial $F(x)=f(x)+\phi(x)$ is of degree $n-1$. Since $n-2$ of its roots are real it can have only real roots, and since $F^{\prime}(y)=0, F(y)=1$, it follows that $\max _{-1 \leqq x \leqq 1} F(x)=1$. Thus $F(x)$ satisfies (1) (obviously $F(x) \neq 0$ for $-1<x<1$ ) and $F(x) \geqq f(x)$ in $-1,+1$, equality occurring only for $-1, y,+1$. Thus $c_{F}>c_{f}$. Hence we may suppose that for $i>2$ all the $x_{i}$ are of the same sign; without loss of generality we may suppose them negative. Suppose that

$$
f(a) f(b)=d^{2}, \quad b-a=c_{f} .
$$

We can suppose that $-1<a<y<b<1$. We now prove that

$$
b-y<y-a \text {. }
$$

For if not then

$$
\left|f^{\prime}(b)\right|>\left|f^{\prime}(a)\right|, \quad f(b)<f(a)
$$

that is,

$$
\begin{array}{r}
\left|f^{\prime}(b)\right|=\left|(b-y) \prod_{i=1}^{n-2}\left(b-y_{i}\right)\right|, \quad\left|f^{\prime}(a)\right|=\left|(y-a) \prod_{i=1}^{n-2}\left(y_{i}-a\right)\right|, \\
y>y_{i}, \quad i=1,2, \cdots, n-2,
\end{array}
$$

where $b-y \geqq y-a$ and all other factors in $\left|f^{\prime}(b)\right|$ are greater then the corresponding factors in $f^{\prime}(a)$. This proves the first inequality of (6). To prove the second inequality we remark that from what has just been said it follows that for $u_{1}-y=y-u_{2},-1<u_{2}<y<u_{1}<1$, we have

$$
\left|f^{\prime}\left(u_{1}\right)\right|>\left|f^{\prime}\left(u_{2}\right)\right|
$$

and since $b-z \geqq y-a$ the second inequality follows by integration.

By simple calculation it follows from (6) that

$$
f(b-\epsilon) f(a-\epsilon)>f(a) f(b)=d^{2}, \quad \epsilon>0 \text { sufficiently small. }
$$

Thus $b-a<c_{f}$. This contradiction proves (5).

Let $x_{n}$ be the root of $f(x)$ with greatest absolute value. Consider the new polynomial

$$
f_{1}(x)=c \frac{x-x_{n}^{\prime}}{x-x_{n}} f(x), \quad x_{n}^{\prime}=x_{n}-\delta, \delta>0
$$


where $c$ is chosen in such a way that $\max _{-1 \leqq x \leqq 1} f_{1}(x)=1$. Then we prove

$$
c_{f_{1}}>c_{f}
$$

To show (7) it will suffice to show that $c_{f}$ is an increasing function of $\left|x_{n}\right|$. Choose $\delta$ so small that if we denote by $y^{(1)}$ the root of $f_{1}^{\prime}(x)$ in $(-1,+1)$ we have $b-y_{1}<y_{1}-a$ (it is clear that $\left.y_{1}<y\right)$.

Put now

$$
c=\left|\frac{1}{f\left(y_{1}\right)} \frac{x_{n}-y_{1}}{x_{n}-\delta-y_{1}}\right| .
$$

(Evidently $c f_{1}(x)$ satisfies (1).)

Now

$$
\begin{aligned}
c^{2} f_{1}(a) f_{1}(b) & =c^{2} \frac{a-x_{n}+\delta}{a-x_{n}} \frac{b-x_{n}+\delta}{b-x_{n}} f(a) f(b) \\
& >\left(1+\frac{\delta}{a-x_{n}}\right)\left(1+\frac{\delta}{b-x_{n}}\right)\left(\frac{1}{1+\delta / y_{1}-x_{n}}\right)^{2} f(a) f(b)
\end{aligned}
$$

(that is, $\left.f\left(y_{1}\right)<1\right)$. But from (5) we have

$$
\delta\left(\frac{1}{a-x_{n}}+\frac{1}{b-x_{n}}\right)>\frac{2 \delta}{\left(\frac{a+b}{2}-x_{n}\right)}>\frac{2 \delta}{y_{1}-x_{n}}
$$

and

$$
\frac{\delta^{2}}{\left(a-x_{n}\right)\left(b-x_{n}\right)}>\frac{\delta^{2}}{\left(\frac{a+b}{2}-x_{n}\right)^{2}}>\frac{\delta^{2}}{\left(y_{1}-x_{n}\right)^{2}} .
$$

Thus

$$
c^{2} f_{1}(a) f_{1}(b)>f(a) f(b)=d^{2} .
$$

Hence (7) is proved.

If $\left|x_{n}\right|$ tends to infinity $f(x)$ tends to $F(x)=f(x) /\left(x-x_{n}\right)$, which is of degree $n-1$. From (7) it follows that $c_{F}>c_{f}$, which proves the theorem.

Let $f(x)$ be a polynomial of degree $n$ all the roots of which are in the interval $(-1,+1)$; and further let $\max _{-1 \leqq x \leqq 1}|f(x)|=1$. For which polynomial is

$$
\int_{-1}^{+1}|f(x)|
$$


maximal? I was not able to answer this question but it seems very likely that the maximum is reached for $f(x)=T_{n}(x / c)$, where $c=1 / x_{n}$, and $x_{n}$ is the greatest root of $T_{n}(x)$ (the $n$th Tchebicheff polynomial). Hence $T_{n}(1 / c)=T_{n}(-1 / c)=0$ and all other roots of $T_{n}(x / c)$ are in $(-1,+1)$. It is easy to see that $T_{n}(x)$ satisfies the following condition: Let $x_{i}$ and $x_{i+1}$ be two consecutive roots of $T_{n}(x)$; then

$$
\frac{1}{x_{i+1}-x_{i}} \int_{x_{i}}^{x_{i+1}}\left|T_{n}(x)\right|=d_{n},
$$

where $d_{n}$ is independent of $i$, and $\lim d_{n}=2 / \pi$.

This fact suggests the following conjecture which is a generalization of the previous one: Let $f(x)$ be a polynomial of degree $n$ all the roots of which are in $(-1,+1)$, such that $\max _{-1 \leqq x \leqq 1}|f(x)|=1$ and let $x_{i}$ and $x_{i+1}$ be two consecutive roots of $f(x)$; then

$$
\int_{x_{i}}^{x_{i+1}}|f(x)| \leqq d_{n}\left(x_{i+1}-x_{i}\right) .
$$

Equality holds only for $T_{n}(c x)$.

It seems very likely that the following result holds: Let $\phi(\theta)$ be a trigonometric polynomial all the roots of which are real, further let $\max _{0 \leqq \theta \leqq 2 \pi}|\phi(\theta)|=1$. Then

$$
\int_{0}^{2 \pi}|\phi(\theta)| \leqq 4 .
$$

Let $f(x)$ be a polynomial of degree $n$ with leading coefficient 1 and all roots in $(-1,+1)$; then the sum of the intervals in $(-1,+1)$ for which $|f(x)| \geqq 1$ does not exceed 1 . The proof is quite simple. Evidently

$$
f(x) f(-x)=\prod_{i=1}^{n}\left(x_{i}^{2}-x^{2}\right) \leqq 1 \quad \text { for }|x| \leqq 1,
$$

equality occurring only for $x=0,\left|x_{i}\right|=1$. Thus one of the numbers $f(x)$ or $f(-x)$ is less than 1 , which establishes the result. It is also easy to see that if the sum of the intervals in question is exactly 1 then $f(x)=(1 \pm x)^{n}$. It would not be difficult to prove the following slightly more general result: Let $f(x)$ have leading coefficient 1 and all roots in $(-1,+1)$; then if $-1<a<0<b<1$ at least one of the numbers $|f(a)|$ or $|f(b)|$ is less than 1 . These problems become very much more difficult if instead of the interval $-1,+1$ we consider the unit circle. The question would be to determine the polynomial (or poly- 
nomials) of degree not greater than $n$ with leading coefficient 1 and all roots in the unit circle such that the area of the set of points for which $|f(x)| \geqq 1$ shall be as big as possible. A first guess would be $f(x)=(x-a)^{n},|a|=1$, but it can be shown that for sufficiently large $n$ this is not the case. The complete solution of this problem seems difficult.

Mr. Eröd ${ }^{2}$ proved that there exists a constant $c$ independent of $n$ such that for a polynomial of degree $n$ satisfying the above conditions the area of the set of points for which $|f(x)| \leqq 1$ is not less than $c$. The best value of $c$ is not known.

\section{Institute for Advanced Study}

${ }^{2}$ Oral communication. 